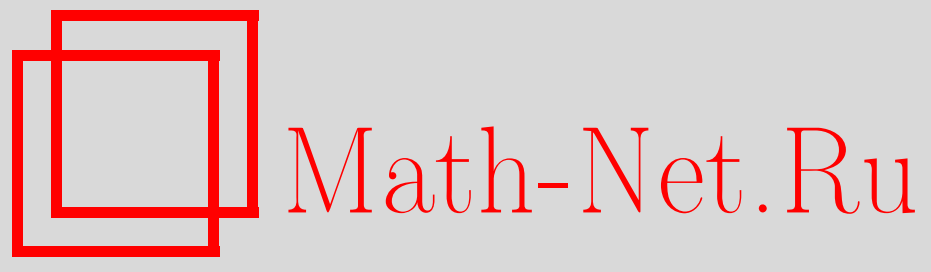

Н. А. Тюрин, Торы Чеканова и псевдоторические структуры, УМH, 2011, том 66, выпуск 1, 185-186

DOI: https://doi.org/10.4213/rm9394

Использование Общероссийского математического портала Math-Net.Ru подразумевает, что вы прочитали и согласны с пользовательским соглашением http://www . mathnet.ru/rus/agreement

Параметры загрузки:

IP: 3.89 .185 .249

26 апреля 2023 г., 10:45:35

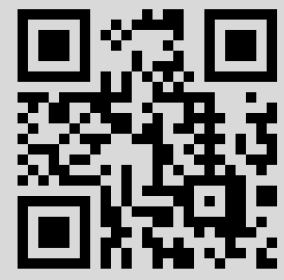




\section{Торы Чеканова и псевдоторические структуры}

\section{Н. А. Тюрин}

Торы Чеканова доставляют примеры экзотических монотонных лагранжевых торов, не переводимых в стандартные торы симплектоморфизмами, в $\mathbb{R}^{2 n}, \mathbb{C P}^{1} \times \cdots \times \mathbb{C P}^{1}$ и некоторых проективных пространствах (см. [1]). Цепочка этих примеров вырастает из конструкции лагранжева тора $\Theta \subset \mathbb{R}^{4}$, представленного явно и неявно в ставших классическими работах [2] и [3]. С другой стороны, в работах [4], [5] было предложено обобщение понятия торической структуры на симплектических многообразиях, получившее название псевдоторической структуры. Так же, как и торическая, псевдоторическая структура порождает пространство лагранжевых слоений с общим гладким слоем, особые слои которых отличаются существованием на них сепаратрисных решений.

В настоящем кратком сообщении мы строим тор Чеканова $\Theta^{k} \subset \mathbb{R}^{2 k-2}$, определенный в [1], как гладкий лагранжев тор, порождаемый псевдоторической структурой. Отсюда вытекает возможность построения экзотических монотонных лагранжевых торов типа Чеканова на торических многообразиях Фано.

Рассмотрим $\mathbb{R}^{2 k+2}$ со стандартными комплексной и симплектической структурами, т. е. $\mathbb{C}^{k+1}$. Зафиксируем координаты $\left(z_{1}, \ldots, z_{k+1}\right)$; тогда имеется отображение $\psi: \mathbb{C}^{k+1} \rightarrow \mathbb{C}$, задаваемое формулой

$$
\left(z_{1}, \ldots, z_{k+1}\right) \mapsto a=z_{1} \times \cdots \times z_{k+1} \in \mathbb{C}_{a} .
$$

Слои $\psi$ - гиперповерхности в $\mathbb{C}^{k+1}$, гладкие за исключением слоя над нулем. Гамильтоново действие тора $T^{k+1}$ может быть выбрано так, что имеется подтор $T_{0}^{k}$, действие которого сохраняет слои $\psi$. А именно, этот подтор выделен условием

$$
\sum_{i=1}^{k+1} \alpha_{i}=0
$$

где $\alpha_{i}$ - веса. Отображения моментов для гамильтонова действия $T_{0}^{k}$ даются средними значениями $F_{i}=\left\langle A_{i} \psi ; \psi\right\rangle, \psi \in \mathbb{C}^{k+1}$, самосопряженных операторов $A_{i}=$ $\operatorname{diag}\left(\lambda_{1}, \ldots, \lambda_{k+1}\right)$, где $\lambda_{i}=1, \lambda_{i+1}=-1$ и $\lambda_{j}=0$ для остальных индексов. Тогда нетрудно видеть, что $\left\{F_{1}, \ldots, F_{k}\right\}$ - коммутирующий набор на $\mathbb{C}^{k+1}$, ограничение которого на каждый гладкий слой $\psi$ есть вполне интегрируемая система (см. [6]). Структура такого типа называется псевдоторической структурой (детальное определение см. в [5]).

Если зафиксировать набор $\left(c_{1}, \ldots, c_{k}\right)$ некритических значений для ограничений $F_{1}^{a}, \ldots, F_{k}^{a}$ на произвольный гладкий слой $N_{a}$ (т. е. $-1<c_{i}<1$ ), то совместное множество уровня $S_{\left(c_{1}, \ldots, c_{k}\right)}^{a}=\left\{F_{i}^{a}=c_{i}\right\} \subset N_{a}$ - гладкий лагранжев тор для каждого $a \neq 0$. Тогда выбор произвольной гладкой петли $\gamma \subset \mathbb{C}_{a}^{*}=\mathbb{C}_{a} \backslash\{0\}$ определяет гладкий лагранжев тор в $\mathbb{C}^{k+1}$ :

$$
T^{k+1}=S_{\gamma,\left(c_{1}, \ldots, c_{k}\right)}=\bigcup_{a \in \gamma} S_{\left(c_{1}, \ldots, c_{n}\right)}^{a} .
$$

Различаются два случая: стандартный тип, когда $\gamma$ нестягиваема в $\mathbb{C}_{a}^{*}$; тип Чеканова, когда $\gamma$ стягиваема в $\mathbb{C}_{a}^{*}$. Названия типов объясняются следующим утверждением.

Работа выполнена при поддержке Лаборатории алгебраической геометрии ГУ-ВШЭ, грант правительства РФ дог. 11.G34.31.0023, а также грантов РФФИ-НЦНИЛ (грант № 10-01-93113) и программы "Ведущие научные школы" (грант НШ-1987.2008.1). 
Рассмотрим две гладкие петли в $\mathbb{C}_{a}^{*}$ : нестягиваемая петля $\gamma_{1}$ есть просто окружность с центром в нуле; для произвольной гладкой петли $\gamma_{0} \subset D \subset \mathbb{C}$ из сектора

$$
D=\left\{z \in \mathbb{C}: 0<|z|<2,0<\arg z<\frac{2 \pi}{k+1}\right\}
$$

(ср. [1; первая формула в разделе 2]), стягиваемая петля $\gamma_{2}$ есть $\left(\gamma_{0}\right)^{k+1}$.

Теорема. Лагранжевы торы $T_{1}, T_{2} \subset \mathbb{C}^{k+1}$, соответствующие значениям $c_{1}=$ $\cdots=c_{k}=0$ и петлям $\gamma_{1}, \gamma_{2} \subset \mathbb{C}_{a}^{*}$, совпадают со стандартным тором и тором $\Theta^{k}$ соответственно.

Доказательство состоит в прямом вычислении (детали можно найти в [7]).

Далее, для экзотических монотонных торов в $\mathbb{C P}^{1} \times \cdots \times \mathbb{C P}^{1}$ и проективных пространствах, предложенных в [1], также имеются описания в терминах псевдоторических структур на этих многообразиях. Отсюда следует, что произвольное симплектическое многообразие с псевдоторической структурой обладает торами чекановского типа. В работе [5] представлен набросок доказательства существования псевдоторической структуры на произвольном торическом многообразии Фано, откуда следует существование монотонных лагранжевых торов типа Чеканова на произвольном торическом многообразии Фано. Напомним, что в исходном случае торы Чеканова не эквивалентны стандартным лагранжевым торам, индуцируемым торической структурой; поэтому естественно ожидать, что торы чекановского типа в произвольном торическом многообразии Фано также окажутся не эквивалентными слоям отображения действия по модулю симплектоморфизмов. Работой над этой гипотезой мы планируем продолжить наши исследования псевдоторических структур.

\section{Список литературы}

[1] Yu. Chekanov, F. Schlenk, Electron. Res. Announc. Math. Sci., 17 (2010), 104-121; arXiv: abs/1003.5960v1. [2] Yu. V. Chekanov, Math. Z., 223:4 (1996), 547-559. [3] Ya. Eliashberg, L. Polterovich, Geometric topology (Athens, GA, 1993), AMS/IP Stud. Adv. Math., 2, part 1, Amer. Math. Soc., Providence, RI, 1997, 313-327. [4] С. А. Белев, Н. А. Тюрин, Матем. заметки, 87:1 (2010), 48-59; англ. пер.: S. A. Belev, N. A. Tyurin, Math. Notes, 87:1 (2010), 43-51. [5] Н.А. Тюрин, TMФ, 162:3 (2010), 307-333; англ. пер.: N. A. Tyurin, Theoret. and Math. Phys., 162:3 (2010), 255-275. [6] Н. Н. Нехорошев, Функи. анализ и его прил., 28:2 (1994), 67-69; англ. пер.: N. N. Nekhoroshev, Funct. Anal. Appl., 28:2 (1994), 128-129. [7] N. A. Tyurin, Twist tori and pseudotoric structures, arXiv: 1004.2574v1.

\section{Н. А. Тюрин (N. A. Tyurin)}

Объединенный институт ядерных исследований, Лаборатория теоретической физики им. Н. Н. Боголюбова

E-mail: tyurin@tyurin.mccme.ru, ntyurin@thsun1.jinr.ru
Представлено А. Г. Сергеевым Принято редколлегией 03.11.2010 